\title{
Diurnal blood pressure variation, risk categories and antihypertensive treatment
}

\author{
Alejandro de la Sierra ${ }^{1}$, Julián Segura ${ }^{2}$, Manuel Gorostidi ${ }^{3}$, José R Banegas ${ }^{4}$, Juan J de la Cruz ${ }^{4}$ \\ and Luis M Ruilope ${ }^{2}$
}

Ambulatory blood pressure (BP) monitoring is a useful tool aiding diagnostic and management decisions in patients with hypertension. Diurnal BP variation or circadian rhythm adds prognostic value to the absolute BP elevation. The Spanish ABPM Registry has collected information from $>30000$ treated hypertensive patients attended by either primary care physicians or referral specialists. The analysis of BP diurnal variation has allowed the conclusion that nocturnal BP decline is related to the level of risk. Patients with blunted nocturnal dip frequently belong to high- or very high-risk categories and specifically are often older, obese, diabetics or with overt cardiovascular or renal disease. With respect to treatment, the non-dipper profile is more often observed in patients receiving several antihypertensive drug agents, but it does not correlate with the time of drug administration. Among patients receiving only one drug, non-dihydropyridine calcium channel blockers and $\alpha$-blockers are associated with less nocturnal BP decline than other antihypertensive drug classes, even after adjusting for the level of risk. Hypertension Research (2010) 33, 767-771; doi:10.1038/hr.2010.111; published online 8 July 2010

Keywords: ambulatory blood pressure monitoring; cardiovascular risk factors; circadian blood pressure pattern; nocturnal blood pressure dip

\section{INTRODUCTION}

Hypertension is one of the most important challenges for public health systems. This relevance is determined by its high prevalence and its association with the risk of cardiovascular and renal diseases. ${ }^{1-3}$ In 2000, approximately a quarter of the worldwide adult population had hypertension, and this proportion is expected to rise to $29 \%$ in $2025 .{ }^{4}$

The current management of hypertensive patients often does not consider or gives little importance to the biological rhythms inherent to the disease process. The development of techniques for ambulatory blood pressure (BP) measurement has generated a series of questions directly related to the biological rhythms of the cardiovascular system. ${ }^{5}$ In recent years, several evidences highlight the influence of nocturnal BP values and, more specifically, the absence of nocturnal dipping and increased morning surge of BP on the development of target organ damage and increased cardiovascular risk. ${ }^{5}$

Ambulatory BP monitoring techniques have expanded the knowledge regarding the circadian rhythm of BP. Several evidences suggest a relationship between cardiovascular complications such as acute myocardial infarction and cerebrovascular disease with circadian BP changes. ${ }^{6}$ In fact, many studies suggest that patients who do not show an appropriate nocturnal dip in BP can suffer from a variety of disorders associated with increased rates of cardiovascular morbidity and mortality. ${ }^{7-9}$ Ohkubo et al. ${ }^{10}$ have shown that a blunted nocturnal decline in BP was a risk factor for cardiovascular mortality in the general population. In this regard, Cuspidi et al..$^{7}$ have shown that the persistence of a non-dipper pattern is associated with an increased left ventricular mass index, a thicker interventricular septum and a larger diameter of both the left atrium and the aortic root, in a group of 375 previously untreated hypertensive patients. Similarly, nondipper hypertensives show a greater degree of insulin resistance and lower levels of adiponectin, compared with those showing a normal dipping pattern. ${ }^{8}$ These non-dipper hypertensive patients have a more severe impairment of endothelial function as manifested by a reduced ability of endothelium-dependent vasodilation, mediated by a decrease in nitric oxide release. ${ }^{9}$ In fact, reverse dippers show wider pulse pressure at night than any other groups, suggesting the potential role of arterial stiffness as an underlying mechanism of impaired cardiovascular risk. ${ }^{11}$ All these changes determine a worse long-term prognosis of those hypertensives with absence of nocturnal dip in BP. In a meta-analysis including data of 3468 patients from four prospective studies, the dipping pattern and the night-day BP ratio significantly and independently predict mortality and cardiovascular events in hypertensive patients without history of major cardiovascular disease. ${ }^{12}$ In diabetic patients, the loss of the physiological circadian pattern was associated with increased mortality in both type 1 and type $2 .{ }^{13}$

${ }^{1}$ Department of Internal Medicine, Hospital Mutua Terrassa, University of Barcelona, Barcelona, Spain; ${ }^{2}$ Hypertension Unit, Department of Nephrology, Hospital 12 de Octubre, Madrid, Spain; ${ }^{3}$ Department of Nephrology, Hospital Universitario Central de Asturias, Oviedo, Spain and ${ }^{4}$ Department of Preventive Medicine and Public Health, Autonomous University of Madrid, Madrid, Spain

Correspondence: Dr LM Ruilope, Hypertension Unit, Hospital 12 de Octubre, Carretera de Andalucia s/n, Madrid 28041, Spain.

E-mail: Ruilope@ad-hocbox.com

Received 15 April 2010; revised 25 May 2010; accepted 2 June 2010; published online 8 July 2010 
Moreover, there is growing evidence linking early morning rise in BP and increased cardiovascular risk. ${ }^{14}$ Although the mechanisms responsible for this relationship are not well known, several factors contribute to hemodynamic and neurohumoral changes. ${ }^{6,14,15}$ In patients with coronary disease, transiently myocardial ischemia may appear in the first $2 \mathrm{~h}$ after waking. ${ }^{16}$ Similarly, the observation of a group of 1167 patients with ischemic stroke revealed that the onset of the stroke occurred in the early morning hours more frequently than at other times of the day ${ }^{17}$ and that the incidence of stroke is directly related to the magnitude of the morning rise in BP. ${ }^{18}$ Similarly, several meta-analyses confirmed the relationship between higher rates of cardiovascular complications (myocardial infarction ${ }^{19}$ and stroke ${ }^{20}$ ) and the early hours of the morning.

Definitely, the availability of techniques for ambulatory BP measurement has shown other aspects of circadian variability of BP (absence of physiological nocturnal dipping, early morning surge) that must be considered as a decisive factor influencing the development and progression of target organ damage and long-term prognosis. Proper management of these factors could positively contribute to the cardiovascular prognosis of hypertensive patients.

\section{CIRCADIAN BP VARIATION AND RISK CATEGORIES}

As mentioned above, there is a close relationship between circadian BP variation and cardiovascular risk. One of the analyses from the Spanish Society of Hypertension ABPM Registry was a comparison between high-risk and low-to-moderate-risk hypertensive patients. ${ }^{21}$ We identified 6534 (37.9\%) hypertensive subjects as having high or very high added cardiovascular risk and 10885 (62.1\%) with low-tomoderate added risk according to the 2003 ESH-ESC guidelines stratification score. ${ }^{22}$ Prevalence of a non-dipping pattern was $58.7 \%$ in high-risk patients and $47.9 \%$ in hypertensives with a lower risk profile. The difference in the non-dipper prevalence between these subgroups of hypertensives was almost entirely caused by an increased prevalence of risers. These data have been updated for the five categories of risk and are exposed in Figure 1. Similarly, a high prevalence of either a non-dipper or a riser pattern of BP has been reproduced in further analyses from our database performed diabetics, patients with chronic kidney disease or hypertensives with coronary heart disease. Hypertensive diabetics were non-dippers in $64.2 \%$ of cases, being $21.0 \%$ risers. Corresponding data in renal and coronary heart disease patients were 72.1 and $25.8 \%$, and 69.4 and $25.1 \%$, respectively. ${ }^{23}$

A relationship between the level of $\mathrm{BP}$ and the non-dipper pattern has also been reported, according to data from our group ${ }^{21}$

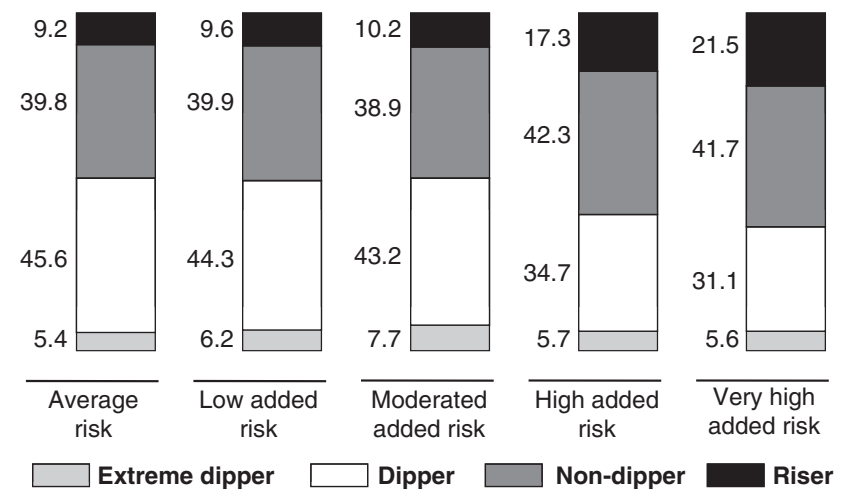

Figure 1 Prevalence of circadian patterns of BP according to cardiovascular risk stratification in 33829 hypertensive patients.
(Table 1). Patients showing most elevated ambulatory systolic BP $(>155 \mathrm{~mm} \mathrm{Hg})$ showed a prevalence of non-dippers of $\approx 70 \%$. However, patients in the lowest levels of ambulatory BP $(<135 \mathrm{~mm} \mathrm{Hg}$ ), but with high-risk showed an increased prevalence of non-dipping compared with those at the same level of BP, but with a lower risk (56.0 vs. $45.7 \%)$.

Opportunities to compare our results regarding prevalence of circadian patterns with that from other studies are scarce. Cuspidi et al. $^{24}$ reported that the prevalence of non-dippers pattern were $28.5 \%$ in low-risk, $32.6 \%$ in medium-risk and $42.2 \%$ in high-risk hypertensives. These investigators evaluated a series of 580 untreated patients, but office grade $3 \mathrm{BP}$, diabetes or associated clinical conditions were excluded. This is obviously in contrast with our database, in which most of the patients were on treatment, and these specific clinical situations were not excluded.

In a recent study, Redon et al. ${ }^{25}$ have shown that the prevalence of non-dippers in patients with stages 3 or 4 chronic kidney disease was $47 \%$, far below our $72.1 \%$ observation. Possible explanations include an office BP considerably lower in the former study and differences in the methodology for diagnosing the dipping status with regard to the nighttime period definition. ${ }^{21,25}$

In summary, high-risk hypertensives show a remarkable high prevalence of abnormalities in circadian BP variation with a key influence in absolute levels of nighttime and 24-h BP. These observations support the recommendation of a wider use of ABPM in high-risk hypertensive patients. ${ }^{21,26}$

\section{INFLUENCE OF ANTIHYPERTENSIVE DRUG TREATMENT ON DIURNAL BP VARIATION}

The Spanish National ABPM Registry has examined the presence of clinical factors associated with nocturnal BP decline in treated and non-treated hypertensive patients. ${ }^{27}$ We present here the effects of treatment (number of antihypertensive drugs and time of administration) in nocturnal BP decline.

This analysis was performed in 34563 hypertensive patients under antihypertensive treatment who were included in the Spanish National ABPM Registry between June 2004 and December 2006. General characteristics include male sex in 53\%, and a mean age of $60.0 \pm 13.8$ years. Office systolic BP/diastolic BP was $149.5 \pm 19.3$ / $87.8 \pm 11.7 \mathrm{~mm} \mathrm{Hg}$ and 24-h ambulatory BP was $130.6 \pm 14.6$ / $77.0 \pm 19.3 \mathrm{~mm} \mathrm{Hg}$. Mean body mass index was $29.0 \pm 4.7 \mathrm{~kg} \mathrm{~m}^{-2}$. Cardiovascular risk factors included dyslipidemia in $37.5 \%$ of cases, diabetes in $18.7 \%$ and current smoking in $17.2 \%$. History of cardiovascular clinical conditions included coronary heart disease in $6.3 \%$, stroke in $4.1 \%$, congestive heart failure in $2.2 \%$ and chronic renal disease in $1.7 \%$.

In this study, BP was measured at the office with a calibrated mercury sphygmomanometer or a validated semiautomatic

Table 1 Prevalence of non-dipper pattern according to four strata of 24-h systolic BP

\begin{tabular}{lccr}
\hline $\begin{array}{l}\text { 24- } \mathrm{h} \text { systolic } \\
\text { BP stratum }\end{array}$ & $\begin{array}{c}\text { High added risk } \\
\mathrm{n} \mathrm{6534}(37.9 \%)\end{array}$ & $\begin{array}{c}\text { Low-to-moderate added risk } \\
\text { n 10685 (62.1\%) }\end{array}$ & $\mathrm{P}$ \\
\hline$>155 \mathrm{~mm} \mathrm{Hg}$ & $69.0 \%$ & $70.0 \%$ & 0.763 \\
$145-155 \mathrm{~mm} \mathrm{Hg}$ & $62.2 \%$ & $56.7 \%$ & 0.023 \\
$135-145 \mathrm{~mm} \mathrm{Hg}$ & $57.3 \%$ & $49.9 \%$ & $<0.001$ \\
$<135 \mathrm{~mm} \mathrm{Hg}$ & $56.0 \%$ & $45.7 \%$ & $<0.001$
\end{tabular}

Abbreviation: BP, blood pressure.

Comparison between high-risk and low-to-moderate-risk patients. 

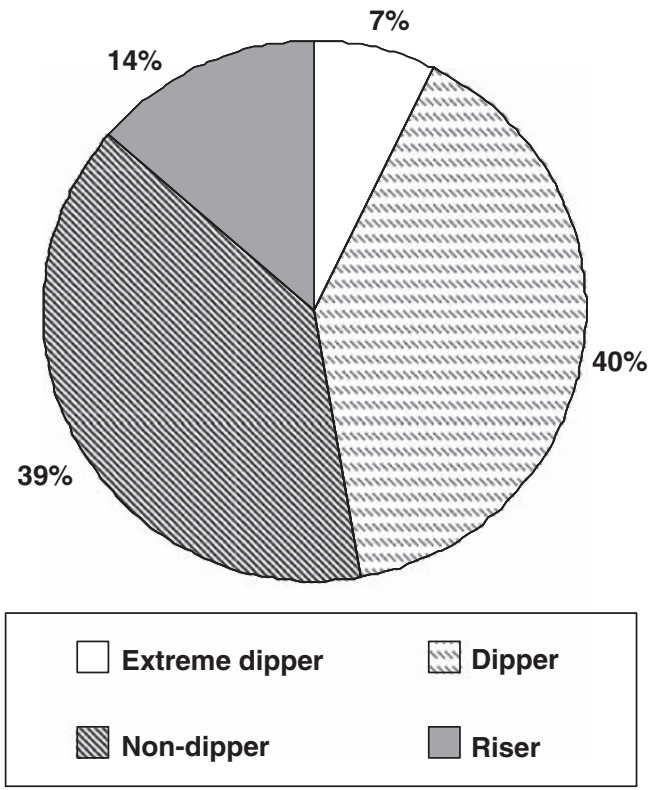

Figure 2 Circadian BP patterns distribution in treated patients from the Spanish ABPM Registry.

oscillometric device, after 5-min rest in a sitting position. BP values were estimated as the mean of two readings. Thereafter, 24-h ABPM was performed using the SpaceLabs 90207 automated non-invasive oscillometric device (Spacelabs Medical, model 90207; Space-Labs Inc., Redmond, WA, USA), programmed to register BP at 20-min intervals for the 24-h period. Patients were instructed to maintain their usual activities, return the following morning for device removal and keep the arm extended and immobile at the time of each cuff inflation. Valid registries had to fulfill a series of pre-established criteria, including $\geqslant 80 \%$ of systolic BPs and diastolic BPs successful recordings during the daytime and nighttime periods, 24-h duration, and at least one BP measurement per hour. Daytime and nighttime periods were defined individually according to the patient selfreported data of going-to-bed and getting-up times. Nocturnal BP decline and circadian patterns were defined by calculating the percent decline in both systolic BP and diastolic BP during the night, using the formula: (daytime BP-nighttime BP)/daytime BP. Normal dipping was diagnosed when BP nocturnal fall was $>10 \%$ (10-20\% dippers and $>20 \%$ extreme dippers). Otherwise, patients were classified as non-dippers ( $0-10 \%$ non-dippers and $<0 \%$ risers).

The distribution of patients among different nocturnal BP fall categories is depicted in Figure 2. Dippers accounted for $39.9 \%$ $(n=13800)$, extreme dippers for $7.2 \%(n=2499)$, non-dippers for $39.4 \%(n=13594)$ and risers for $13.5 \%(n=4670)$. The prevalence of dipping (dippers/extreme dippers) and non-dipping (non-dippers/ risers) patterns was 47.2 and $52.8 \%$, respectively.

The main characteristics of the study sample according to dipping status are shown in Table 2. Dippers and non-dippers showed statistically significant differences in most of the study variables. Those with a non-dipping profile were older, more frequently women and obese, and had a longer duration of hypertension. They also had more frequently a previous diagnosis of dyslipidemia, type 2 diabetes or cardiovascular disease. Conversely, the proportion of smokers was slightly lower in those with a non-dipping pattern.

Influence of antihypertensive therapy in non-dipping status was examined by comparing nocturnal BP decline and proportion of
Table 2 Characteristics of treated hypertensive patient according to dipping (dippers/extreme dippers) and non-dipping (non-dippers/risers) status

\begin{tabular}{lccr}
\hline Variable & $\begin{array}{c}\text { Dipping } \\
(\mathrm{n}=16299)\end{array}$ & $\begin{array}{c}\text { Non-dipping } \\
(\mathrm{n}=18264)\end{array}$ & P-value \\
\hline $\begin{array}{l}\text { Sex (\% women) } \\
\text { Age, year }\end{array}$ & 45.5 & 48.5 & $<0.001$ \\
& $55.7 \pm 13.5$ & $61.9 \pm 13.3$ & $<0.001$ \\
SBP, $m m$ Hg & & & \\
Clinic & & & \\
24-h & $149.1 \pm 18.4$ & $149.8 \pm 20.0$ & 0.003 \\
Daytime & $129.3 \pm 13.1$ & $131.7 \pm 15.7$ & $<0.001$ \\
Nighttime & $134.7 \pm 13.7$ & $132.8 \pm 15.7$ & $<0.001$ \\
$\quad \%$ Nocturnal decline in BP, $\mathrm{mm} \mathrm{Hg}$ & $113.6 \pm 12.3$ & $128.6 \pm 16.8$ & $<0.001$ \\
& $15.6 \pm 4.2$ & $3.1 \pm 6.1$ & $<0.001$
\end{tabular}

DBP, $m m \mathrm{Hg}$
Clinic
$24-\mathrm{h}$
Daytime
Nighttime
$\quad \%$ Nocturnal decline in BP, mm Hg
$24 \mathrm{~h} \mathrm{BP}<130 / 80, \%$
Duration of hypertension, year
BMI, kg ${ }^{-2}$
Current smoking, \%
Dyslipidemia, \%
Diabetes, \%
History of cardiovascular disease, \%
Congestive heart failure
Cerebrovascular disease
Coronary heart disease
Chronic renal disease

$\begin{array}{ccr}89.0 \pm 11.1 & 86.6 \pm 12.1 & <0.001 \\ 78.2 \pm 9.7 & 76.2 \pm 10.6 & <0.001 \\ 82.3 \pm 10.1 & 77.8 \pm 10.9 & <0.001 \\ 65.7 \pm 9.1 & 71.6 \pm 10.7 & <0.001 \\ 20.2 \pm 5.7 & 7.8 \pm 7.1 & <0.001 \\ 42.5 & 41.5 & 0.057 \\ 4.9 \pm 6.4 & 6.9 \pm 7.5 & <0.001 \\ 28.6 \pm 4.5 & 29.3 \pm 4.8 & <0.001 \\ 19.5 & 15.1 & <0.001 \\ 35.2 & 39.5 & <0.001 \\ 14.5 & 22.4 & <0.001\end{array}$

Abbreviations: BMI: body mass index; BP, blood pressure; $\mathrm{DBP}$, diastolic blood pressure SBP, systolic blood pressure.

dippers in patients depending on the number of antihypertensive drugs used. Figure 3 (left) shows the proportion of dipping and non-dipping patterns in groups of patients treated with monotherapy, two antihypertensive drugs or three or more drugs. As observed, the proportion of dippers increased as the number of drugs was greater. Moreover, as also observed in Figure 3 (right), nocturnal BP decline was also less pronounced as the number of drugs increased. In multivariate models, the number of antihypertensive drugs was associated with both the amount of BP decrease during the night $(\beta$ : $-0.700 ; 95 \%$ confidence interval $(\mathrm{CI}):-0.812$ to -0.588 ; $P<0.001$ ) and the classification into the non-dipper status (odds ratio: 1.22 ; $95 \%$ CI: $1.15-1.29 ; P<0.001)$.

The relationship of the time of the day when treatment was administered with diurnal BP variation was also examined. A majority of the patients (26410) received antihypertensive treatment only in the morning, whereas less patients received antihypertensive treatment only at night (3646) or combined morning and night (3649). We did not find differences between dippers and non-dippers in the proportion of patients receiving all or part of their medication at bedtime (20.5 vs. 21.2\%; $P=0.352$ ). Differences in nocturnal BP decline were minimal, but with statistical significance. The nocturnal BP decline (systolic/diastolic) was $8.2 / 12.8$ in those treated only in the morning, 8.0/12.5 in those treated only at bedtime and 7.5/12.2 in those treated both in the morning and at bedtime $(P=0.004$ for systolic and $P=0.012$ for diastolic, respectively). However, the time 

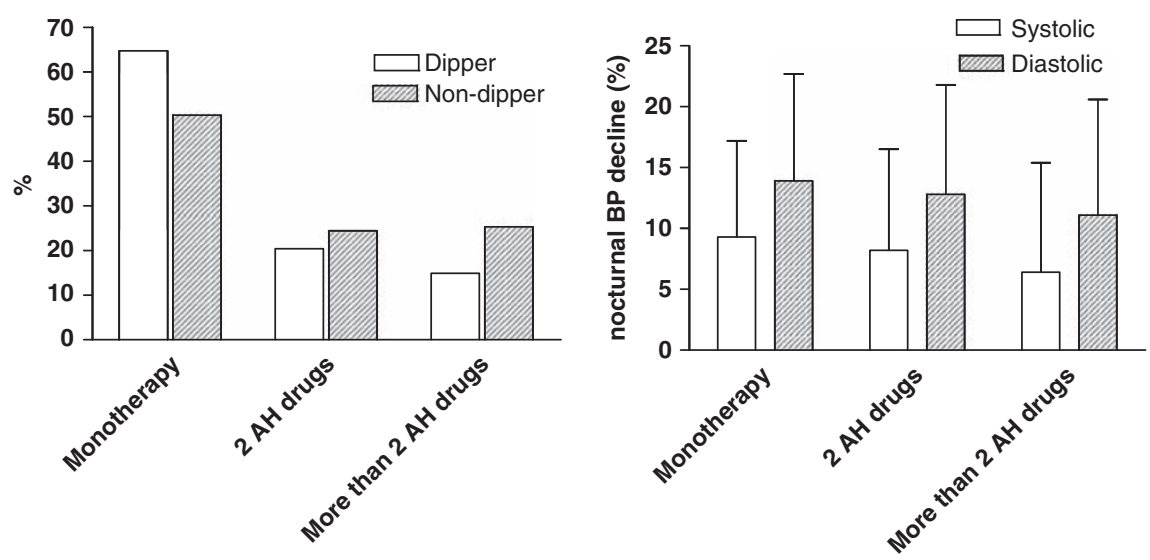

Figure 3 Left: proportion of dippers and non-dippers depending on the number of antihypertensive drugs used $(P<0.001)$. Right: nocturnal $\mathrm{BP}$ fall (systolic and diastolic) in patients depending on the number of antihypertensive drugs used $(P<0.001)$.

when treatment was administered was strongly influenced by the risk profile of the patient. Thus, patients who received all or part of their antihypertensive medication at bedtime were older, more frequently diabetics and had higher BP values. In the multivariate analyses, time of administration was not associated with either the dipping pattern or the amount of BP decline during the night.

In summary, using a large cohort of patients from the ABPM Spanish database we have observed that the amount of nocturnal BP decline was closely related to the level of risk. ${ }^{27}$ Non-dipping was associated with advanced age, obesity and diabetes, conditions that increase cardiovascular risk. Moreover, a history of previous cardiovascular or renal disease was more common in non-dippers than in dippers. With respect to antihypertensive treatment, we have previously reported that treated patients had a blunted nocturnal BP decline with respect to those untreated. ${ }^{27}$ This has been attributed to the use of antihypertensive treatment, which is commonly administered in the morning and lowers BP especially during daytime. ${ }^{28,29}$ With our data, it seems to be an inaccurate assumption. We observed that the proportion of patients receiving all or a part of their medication at night was not different between dippers and nondippers. Moreover, although some differences in the nocturnal BP decline were observed depending on the time when antihypertensive treatment was administered, these differences can be explained by the level of risk of patients, and they disappeared in the multivariate analysis. ${ }^{30,31}$ In support of this, the need for more antihypertensive drugs in the non-dipping group could also reflect the severity and the difficulty to treat elevated BP.

\section{TYPE OF ANTIHYPERTENSIVE DRUG AND DIURNAL BP VARIATION}

Table 3 shows the proportion of dippers and non-dippers, as well as the systolic and diastolic nocturnal BP dip in the cohort of 34563 treated hypertensives depending on the antihypertensive drug class used for treatment in monotherapy. As observed, those who received either non-dihydropyridine calcium channel blockers or $\alpha$-blockers presented a blunted nocturnal dip and higher proportion of non-dippers. This relationship was also present in the multiple linear regression analysis using nocturnal systolic ( $\beta$ : $0.035 ; \quad 95 \%$ CI: $0.022-0.047$ ) or diastolic ( $\beta$ : $0.039 ; 95 \%$ CI: $0.026-0.053$ ) BP decline as dependent variable. Moreover, these results were also observed in the logistic regression analysis (odds ratio: 1.01 ; 95\% CI: 1.00-1.02).
Table 3 Nocturnal BP fall (systolic and diastolic) and prevalence of non-dipping in hypertensives treated with specific drug classes in monotherapy

\begin{tabular}{lccc}
\hline AH drug class & Systolic BP fall & Diastolic BP fall & \% of non-dippers \\
\hline Diuretics (3417) & $9.3 \pm 7.8$ & $14.2 \pm 8.6$ & 52.0 \\
B-Blockers (2626) & $9.0 \pm 8.1$ & $13.6 \pm 8.9$ & 53.1 \\
DHPCCB (1424) & $7.9 \pm 7.6$ & $12.5 \pm 8.6$ & 60.2 \\
NDHPCCB (240) & $6.2 \pm 8.6$ & $10.3 \pm 8.8$ & 66.2 \\
ACEI (3174) & $9.4 \pm 7.9$ & $13.8 \pm 8.8$ & 52.1 \\
ARB (2631) & $9.1 \pm 8.4$ & $13.7 \pm 8.9$ & 52.1 \\
$\alpha$-Blockers (410) & $6.2 \pm 8.2$ & $10.6 \pm 8.4$ & 70.1 \\
\hline
\end{tabular}

Abbreviations: ACE, angiotensin-converting enzyme inhibitors; $A H$, antihypertensive; $\mathrm{ARB}$, angiotensin receptor blocker; $\mathrm{BP}$, blood pressure; $\mathrm{DHPCCB}$, dihydropyridine calcium channel blockers; NDHPCCB, non-dihydropyridine calcium channel blockers.

These results need to be interpreted with caution, as they do not represent a prospective controlled trial, just only observational data. The use of specific drug classes is related to the clinical profile of the patients and this profile has great influence on the circadian pattern as described above. Moreover, grouping antihypertensive drug classes does not take into account particular pharmacokinetic and pharmacodynamic properties of the member belonging to the same class.

In summary, the Spanish ABPM Registry, the largest database on ABPM in the world, has provided evidence regarding the circadian patterns of the population of treated hypertensives. The non-dipping pattern is very common and closely related to the level of risk of the patient. In addition to this, and even understanding that this level of risk is a powerful determinant of treatment, it seems that a more intense antihypertensive treatment, with two or more drugs, and the use of some specific drug classes, such as non-dihydropyridine calcium channel blockers or $\alpha$-blockers is associated with a nondipping pattern, a finding that needs to be corroborated in specifically designed prospective trials.

1 Neaton JD, Wentworth D. Serum cholesterol blood pressure, cigarette smoking, and death from coronary heart disease. Overall findings and differences by age for 316.099 white men. Multiple Risk Factor Intervention Trial Research Group. Arch Intern Med 1992; 152: 56-64. 
2 Prospective Studies Collaboration. Age-specific relevance of usual blood pressure to vascular mortality: a meta-analysis of individual data for one million adults in 61 prospective studies. Lancet 2002; 360: 1903-1913.

3 Weiner DE, Tighiouart H, Amin MG, Stark PC, MacLeod B, Griffith JL, Salem DN, Levey AS, Sarnak MJ. Chronic kidney disease as a risk factor for cardiovascular disease and all-cause mortality: a pooled analysis of community-based studies. J Am Soc Nephrol 2004; 15: 1307-1315.

4 Kearney PM, Whelton M, Reynolds K, Muntner P, Whelton P, He J. Global burden of hypertension: analysis of worldwide data. Lancet 2005; 365: 217-223.

5 Hassler C, Burnier M. Circadian variations in blood pressure: implications for chronotherapeutics. Am J Cardiovasc Drugs 2005; 5: 7-15.

6 Giles T. Relevance of blood pressure variation in the circadian onset of cardiovascular events. J Hypertens 2005; 23(Suppl 1): S35-S39.

7 Cuspidi C, Meani S, Salerno M, Valerio C, Fusi V, Severgnini B, Lonati L, Magrini F, Zanchetti A. Cardiovascular target organ damage in essential hypertensives with or without reproducible nocturnal fall in blood pressure. J Hypertens 2004; 22: 273-280.

8 Della Mea P, Lupia M, Bandolin V, Guzzon S, Sonino N, Vettor R, Fallo F. Adiponectin, insulin resistance, and left ventricular structure in dipper and nondipper essential hypertensive patients. Am J Hypertens 2005; 18: 30-35.

9 Higashi Y, Nakagawa K, Kimura M, Noma K, Hara K, Sasaki S, Goto C, Oshima T, Chayama K, Yoshizumi M. Circadian variation of blood pressure and endothelial function in patients with essential hypertension: a comparison of dippers and nondippers. J Am Coll Cardiol 2002; 40: 2039-2043.

10 Ohkubo T, Hozawa A, Yamaguchi J, Kikuya M, Ohmori K, Michimata M, Matsubara M, Hashimoto J, Hoshi H, Araki T, Tsuji I, Satoh H, Hisamichi S, Imai Y. Prognostic significance of the nocturnal decline in blood pressure in individuals with and without high 24-h blood pressure: the Ohasama study. J Hypertens 2002; 20: 2183-2189.

11 Jerrard-Dunne P, Mahmud A, Feely J. Circadian blood pressure variation: relationship between dipper status and measures of arterial stiffness. J Hypertens 2007; 25: 1233-1239.

12 Fagard RH, Thijs L, Staessen JA, Clement DL, De Buyzere ML, De Bacquer DA. Nightday blood pressure ratio and dipping pattern as predictors of death and cardiovascular events in hypertension. J Hum Hypertens 2009; 23: 645-653.

13 Sturrock ND, George E, Pound N, Stevenson J, Peck GM, Sowter H. Non-dipping circadian blood pressure and renal impairment are associated with increased mortality in diabetes mellitus. Diabet Med 2000; 17: 360-364.

14 Weber MA. The 24-h blood pressure pattern: does it have implications for morbidity and mortality? Am J Cardiol 2002; 89(Suppl 2A): 27A-33A.

15 Kario K. Morning surge and variability in blood pressure: a new therapeutic target? Hypertension 2005; 45: 485-486.

16 Rocco MB, Barry J, Campbell S, Nabel E, Cook EF, Goldman L, Selwyn AP. Circadian variation of transient myocardial ischemia in patients with coronary artery disease. Circulation 1987; 75: 395-400.

17 Marler JR, Price TR, Clark GL, Muller JE, Robertson T, Mohr JP, Hier DB, Wolf PA, Caplan LR, Foulkes MA. Morning increase in onset of ischemic stroke. Stroke 1989; 20: $473-476$.
18 Kario K, Pickering TG, Umeda Y, Hoshide S, Hoshide Y, Morinari M, Murata M, Kuroda T, Schwartz JE, Shimada K. Morning surge in blood pressure as a predictor of silent and clinical cerebrovascular disease in elderly hypertensives: a prospective study. Circulation 2003; 107: 1401-1406.

19 Cohen MC. Meta-analysis of the morning excess of acute myocardial infarction and sudden cardiac death. Am J Cardiol 1997; 79: 1512-1516.

20 Elliott WJ. Circadian variation in the timing of stroke onset - a meta-analysis. Stroke 1998; 29: 992-996.

21 Gorostidi M, Sobrino J, Segura J, Sierra C, De la Sierra A, Hernandez del Rey R, Vinyoles E, Galcerán JM, López-Eady MD, Marín R, Banegas JR, Sarría A, Coca A, Ruilope LM. Ambulatory blood pressure monitoring in hypertensive patients with high cardiovascular risk: a cross-sectional analysis of a 20 000-patient database in Spain. J Hypertens 2007; 25: 977-984.

22 Guidelines Committee. 2003 European Society of Hypertension-European Society of Cardiology guidelines for the management of arterial hypertension. J Hypertens 2003; 21: 1011-1053.

23 Gorostidi M, González-Albarrán O Segura J, De la Sierra A, Sobrino J, de la Cruz JJ, Comerma J, De Miguel A, Martinez-Escudero MV, Roca-Fusalba A, Pacho B, Almarcha $\mathrm{N}$, Banegas JR, Aranda P, Ruilope LM. Prevalence of abnormalities in ambulatory blood pressure monitoring in hypertensive patients with diabetes. J Hypertens 2009; 27(Suppl 4): S239 [abstract].

24 Cuspidi C, Meani S, Valerio C, Fusi V, Zanchetti A. Nocturnal non-dipping pattern in untreated hypertensive at different cardiovascular risk according to the $2003 \mathrm{ESH} / \mathrm{ESH}$ guidelines. Blood Press 2006; 15: 37-44.

25 Redon J, Plancha E, Swift PA, Pons S, Muñoz J, Martinez F. Nocturnal blood pressure and progression to end-stage renal disease or death in nondiabetic chronic kidney disease stages 3 and 4. J Hypertens 2010; 28: 602-607.

26 Parati G, Ibsen H. Twenty-four-hour ambulatory blood pressure profiles of high-risk patients in general practice: data from an ambulatory blood pressure monitoring registry. J Hypertens 2007; 25: 929-933.

27 De la Sierra A, Redon J, Banegas JR, Segura J, Parati G, Gorostidi M, de la Cruz JJ, Sobrino J, Llisterri JL, Alonso J, Vinyoles E, Pallarés V, Sarría A, Aranda P, Ruilope LM. Prevalence and factors associated with circadian blood pressure patterns in hypertensive patients. Hypertension 2009; 53: 466-472.

28 Fagard RH, Celis H, Thijs L, Staessen JA, Clement DL, De Buyzere ML, De Bacquer DA. Daytime and nighttime blood pressure as predictors of death and cause-specific cardiovascular events in hypertension. Hypertension 2008; 51: 55-61.

29 Staessen JA, Thijs L, Fagard R, O'Brien ET, Clement D, de Leeuw PW, Mancia G, Nachev C, Palatini P, Parati G, Tuomilehto J, Webster J. Predicting cardiovascular risk using conventional vs ambulatory blood pressure in older patients with systolic hypertension. JAMA 1999; 282: 539-546.

30 De la Sierra A, Banegas JR, Redon J, Segura J, Gorostidi M, Ruilope LM. Response to nondipping in patients with hipertensión. Hypertension 2009; 53: e36 [letter].

31 De la Sierra A, Banegas JR, Redon J, de la Cruz JJ, Segura J, Gorostidi M, Ruilope LM. Response to timing of antihypertensive therapy and circadian blood pressure patterns. Hypertension 2009; 53: e42 [letter]. 